\title{
Einwirkung von Chlorwasserstoff auf Invertase.
}

II. Mitteilung.

Von

Theodor Panzer.

(Der Redaktion zugegangen am 18. Oktober 1912.)

Die im folgenden beschriebenen Versuche sind Parallelversuche zu meinen Untersuchungen über die Einwirkung von gasförmigem Chlorwasserstoff auf trockene Diastase. ${ }^{1}$ ) Sie sollten lehren, ob auch ein anderes Ferment dasselbe Verhalten gegen Chlorwasserstoff zeigt, wie die Diastase. Diese Versuche wurden auch genau in derselben Weise angestellt, wie die Versuche mit Diastase, mit Ausnahme jener Abänderungen, welche durch die andere Wirkungsweise der Invertase bedingt sind.

Im großen und ganzen sind die Resultate hier ähnlich wie bei der Diastase, doch bestehen einige wesentliche Unterschiede. Die Hauptresultate, in kurzen Worten zusammengefaßt, sind folgende:

Auch das Invertasepräparat nimmt beträchtliche Mengen von Chlorwasserstoff auf und zwar noch größere Mengen als die Diastasepräparate, und verliert dadurch seine Wirksamkeit. Im Vakuum gibt es gleichfalls einen großen Teil des aufgenommenen Chlorwasserstoffs ab, erlangt aber dadurch seine Wirksamkeit nicht wieder.

\section{Verwendetes Präparat.}

Das Invertasepräparat, welches zu meinen Versuchen verwendet worden ist, war nach dem Verfahren von W. A. Osborne ${ }^{2}$ ) aus Hefe dargestellt worden. Bei der Reinigung

1) Diese Zeitschrift, Bd. 82, S. 276.

2) Diese Zeitschrift, Bd. 28, S. 399. 
des Präparates blieb ich indessen auf halbem Wege stehen, um nicht $\mathrm{zu}$ große Verluste zu erleiden. In großen Zügen war das Verfahren folgendes: $10 \mathrm{~kg}$ Hefe wurden nach längerer Behandlung mit Alkohol mit Chloroformwasser ausgezogen. Der filtrierte Auszug wurde mit Alkohol gefällt, der Niederschlag mit Alkohol und Äther gewaschen und getrocknet. Das Gewicht dieses rohen Fermentpräparates betrug $60 \mathrm{~g}$. Das Rohprodukt wurde nun portionenweise mit lauwarmem Wasser angerührt, der filtrierte Auszug nochmals mit Alkohol gefällt, der Niederschlag abermals gewaschen und im Vakuum über Chlorcalcium getrocknet. Der trockene Niederschlag, dessen Gewicht $36 \mathrm{~g}$ betrug, bildete das zu den Versuchen verwendete Fermentpräparat. Es zeigte noch in geringem Maße Eiweißreaktionen und reagierte auf Lackmus ganz schwach sauer.

Dieses Fermentpräparat wurde, sowie es mit den Diastasepräparaten geschehen war, zunächst einer analytischen Untersuchung unterzogen.

Stickst off $0,1755 \mathrm{~g}$ lieferten $8,6 \mathrm{ccm} \mathrm{N}$ bei $18,2^{\circ} \mathrm{C}$. und $741,3 \mathrm{~mm}$, entsprechend $5,64 \% \mathrm{~N}$;

$0,2186 \mathrm{~g}$ lieferten $10,4 \mathrm{ccm} \mathrm{N}$ bei $20,5^{\circ} \mathrm{C}$. und $750,5 \mathrm{~mm}$, entsprechend $5,49 \% \mathrm{~N}$;

$$
\text { im Mittel : } 5,57 \% \mathrm{~N} \text {. }
$$

Asche: $0,2357 \mathrm{~g}$ lieferten $0,0525 \mathrm{~g}$ Asche, entsprechend $22,28 \%$ Asche;

$0,2398 \mathrm{~g}$ lieferten $0,0531 \mathrm{~g}$ Asche, entsprechend $22,14 \%$ Asche ;

im Mittel: 22,21\% Asche.

Amidstickstoff: $0,5344 \mathrm{~g}$ lieferten $21,7 \mathrm{~cm} \mathrm{~N}$ bei $21,0^{\circ} \mathrm{C}$. und $723,3 \mathrm{~mm}$, entsprechend $2,25 \% \mathrm{~N}$;

$04899 \mathrm{~g}$ lieferten $20,4 \mathrm{~cm} \mathrm{~N}$ bei $17,5^{\circ} \mathrm{C}$. und $726,2 \mathrm{~mm}$, entsprechend $2,35 \% \mathrm{~N}$;

$$
\text { im Mittel: 2,30\% } \mathrm{N} \text {. }
$$

Titrierung und Formoltitrierung: $0,4642 \mathrm{~g}$ verbrauchten zur Neutralisation $0,722 \mathrm{ccm}$ Normallauge (entsprechend $155,5 \mathrm{ccm}$ für $100 \mathrm{~g}$ Substanz), weiter nach Zusatz von Formol 1,078 ccm Normallauge, entsprechend 3,25\% N; 
$0,4361 \mathrm{~g}$ verbrauchten zur Neutralisation $0,719 \mathrm{ccm}$ Normallauge (entsprechend $164,9 \mathrm{ccm}$ für $100 \mathrm{~g}$ Substanz, weiter nach Zusatz von Formol $0,963 \mathrm{ccm}$ Normallauge, entsprechend $3,09 \% \mathrm{~N}$;

im Mittel: zur Neutralisation 160,2 ccm Normallauge und 3,17\% formoltitrierbarer Stickstoff.

$\begin{array}{lr}\text { Stickstoff } & 5,57 \% \\ \text { Asche } & 22,21 \% \\ \text { Amidstickstoff } & 2,30 \% \\ \text { Formoltitrierbarer Stickstoff } & 3,17 \%\end{array}$

Mittelwerte.

Acidität entsprechend 160,2 ccm Normallauge für 100g Substanz.

Hervorgehoben sei nur der hohe Aschengehalt des Präparates, ferner der hohe Gehalt an Amidstickstoff und formoltitrierbarem Stickstoffe, der rund etwa die Hälfte des Gesamtstickstoffes beträgt, während diese Zahlen bei den untersuchten Diastasepräparaten nur etwa $1 / 15$ bis $1 / 20$ des Gesamtstickstoffgehaltes ausmachen, endlich die hohe Acidität, welche etwa 5-6 mal so groß ist, wie bei den Diastasepräparaten. Weitere Betrachtungen sollen vorläufig nicht an diese Tatsachen geknüpft werden.

Behandlung mit Chlorwasserst offgas.

Diese Versuche wurden in demselben Apparate und auf dieselbe Weise vorgenommen, wie die Versuche an Diastasepräparaten. Die Resultate sind in folgender Tabelle zusammengestellt. Die Berechnung in Prozenten bedeutet wieder, wie viel Teile Chlorwasserstoff von 100 Teilen Invertasepräparat aufgenommen worden sind.

a) Versuche mit Luftüberleitung bei $50^{\circ} \mathrm{C}$.

\begin{tabular}{c|c|c}
\hline $\begin{array}{c}\text { Verwendete Menge } \\
\text { des Invertasepräparates }\end{array}$ & $\begin{array}{c}\text { Aufgenommene } \\
\text { Chlorwasserstoffmenge } \\
\mathrm{g}\end{array}$ & In Prozenten \\
\hline 0,1453 & 0,0230 & 15,83 \\
0,2476 & 0,0392 & 15,83 \\
0,1712 & 0,0273 & 15,94 \\
0,2481 & 0,0472 & 19,02 \\
\end{tabular}

Hoppe-Seyler's Zeitschrift f. physiol. Chemie. LXXXII. 
b) Versuche mit Luftüberleitung bei Zimmertemperatur.

\begin{tabular}{|c|c|c|}
\hline $\begin{array}{c}\text { Verwendete Menge } \\
\text { des Invertasepräparates } \\
\mathbf{g}\end{array}$ & $\begin{array}{c}\text { Aufgenommene } \\
\text { Chlorwasserstoffmenge } \\
\mathrm{g}\end{array}$ & In Prozenten \\
\hline 0,2044 & 0,0503 & 24,60 \\
\hline 0,4873 & 0,1062 & 21,79 \\
\hline 0,4698 & 0,0890 & 18,94 \\
\hline 0,5103 & 0,1173 & 22,99 \\
\hline 0,4914 & 0,1009 & 20,53 \\
\hline 0,5320 & 0,1163 & 21,86 \\
\hline 0,4940 & 0,1068 & 21,62 \\
\hline
\end{tabular}

Auch die Invertasepräparate sind bei der Behandlung mit Chlorwasserstoff um eine Nuance dunkler geworden und rochen nach Chlorwasserstoff. Gegen feuchte Luft waren sie ebenfalls sehr empfindlich, sie färbten sich dunkel und zerflossen.

Die von dem Invertasepräparate aufgenommenen Chlorwasserstoffmengen sind durchwegs viel größer als diejenigen, welche von den Diastasepräparaten aufgenommen worden sind. Sie zeigen aber auch hier keine Übereinstimmung, was wiederum den Schluß gestattet, daß der Chlorwasserstoff zum Teile leicht zersetzliche Verbindungen, mit gewissen Atomgruppen des Invertasepräparates eingegangen ist.

Nur die drei ersten Versuche, in welchen bei $50^{\circ} \mathrm{C}$. Luft übergeleitet worden ist, zeigen geradezu auffallende Übereinstimmung, es sind dies gleichzeitig die kleinsten Werte der ganzen Reihe. Man könnte sich daher vorstellen, daß bei diesem Werte die Zersetzung der Chlorwasserstoffverbindungen einen gewissen Ruhepunkt erreicht. Wenn der 4. Wert der Versuche mit Erwärmung auf $50^{\circ} \mathrm{C}$. mit den drei anderen Werten nicht übereinstimmt, sondern größer ist, so würde dies nicht mehr bedeuten, als daß in dem 4 . Versuche aus irgendeinem Grunde dieser Ruhepunkt noch nicht erreicht worden ist.

Nimmt man an, daß unter geeigneten Umständen jedes Stickstoffatom des Invertasepräparates ein Molekül Chlorwasserstoff binden kann, so würde dies bei einem Stickstoffgehalte von $5,57 \%$ eine Chlorwasserstoffaufnahme von $14,49 \%$ be- 
deuten. Die gefundenen Zahlen liegen aber hier durchwegs über $14,49 \%$, während sie bei den Diastaseversuchen alle kleiner waren, als der aus dem Stickstoffgehalte berechnete Wert. Dieser Umstand hängt offenbar mit dem hohen Aschengehalte des Invertasegehaltes zusammen. Seine unorganischen Bestandteile werden zweifellos relativ große Chlorwasserstoffmengen binden können. Wie groß diese aber sind, darüber kann man sich ohne genauere Kenntnis der unorganischen Bestandteile kein annäherndes Urteil bilden.

Bei den Diastaseversuchen konnte aus den Resultaten der Formoltitrierung berechnet werden, wieviel Chlorwasserstoff durch Aminogruppen gebunden wird. Die analoge $\mathrm{Be}$ rechnung würde ergeben aus $3,17 \%$ formoltitrierbarem Stickstoff 8,18\% durch Aminogruppen gebundenen Chlorwasserstoff. Dieser Schluß ist jedoch hier nicht gestattet, weil, wie später gezeigt werden wird, die Menge hes formoltitrierbaren Stickstoffs durch die Einwirkung von Chlorwasserstoff erheblich geändert wird.

In der gleichen Weise wie bei den Versuchen mit Diastase wurden auch hier Auspumpversuche angestellt. Sie ergaben folgende Resultate.

In Gewichten:

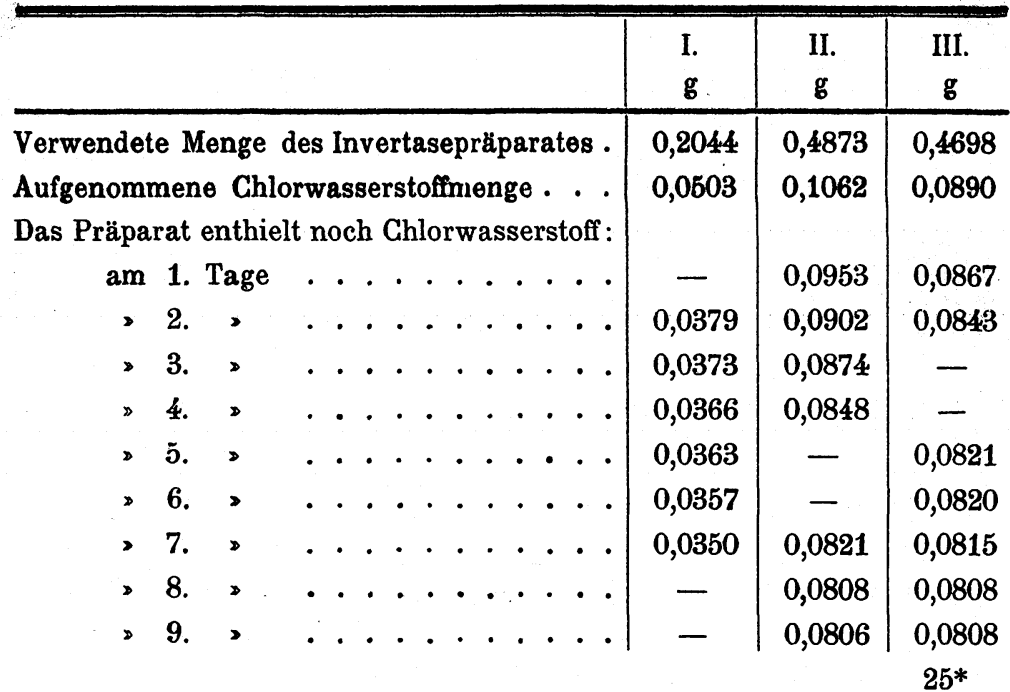


In Gewichten. - Fortsetzung.

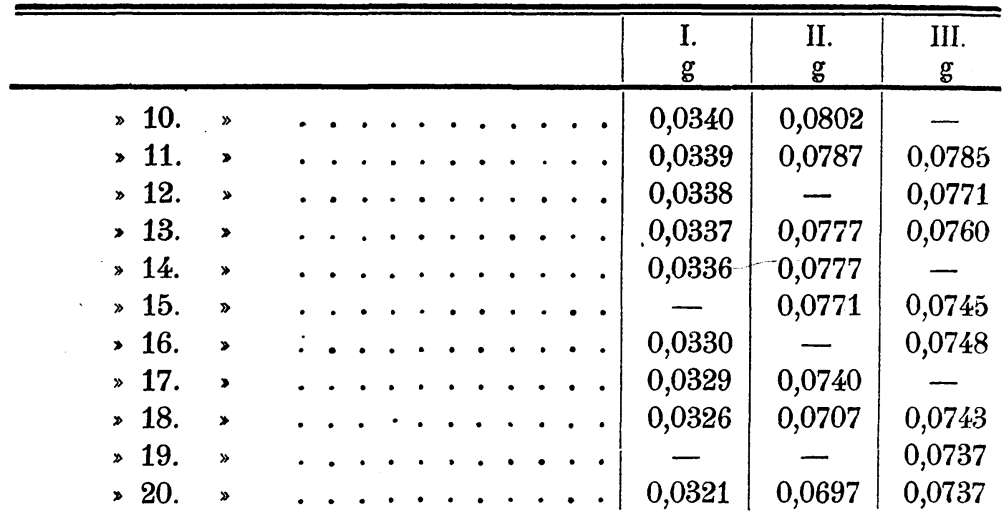

In Prozenten:

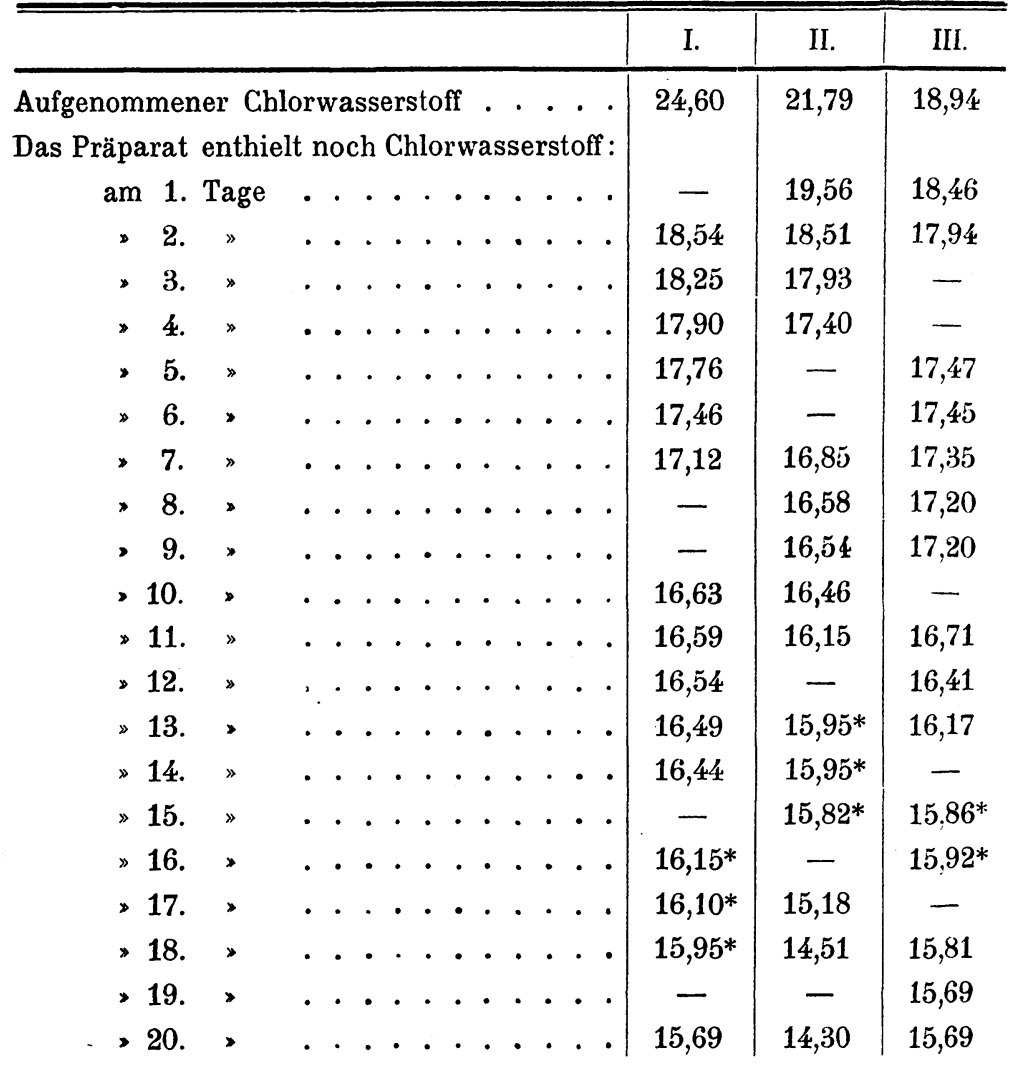


So wie bei den Diastaseversuchen, zeigt sich auch hier eine ständige Abgabe von Chlorwasserstoff, welche, wenigstens in den 20 Versuchstagen, nicht zum Stillstande kommt und allem Anscheine nach bei längerer Fortsetzung der Versuche nicht so bald zum Stillstande kommen dürfte. Die Abgabe geht auch hier wieder in den ersten Tagen sehr rasch vor sich, wird danach recht langsam und nähert sich einer gewissen Gleichmäßigkeit. In graphischer Darstellung würde die Chlorwasserstoffabgabe wieder einer Kurve entsprechen, welche zunächst steil abfällt und dann sich, in großen Zügen betrachtet, ungefähr einer langsam absteigenden Geraden nähert. Die Annäherung des zweiten Abschnittes der Kurve ist hier noch weniger streng als in den Diastaseversuchen, denn in diesem zweiten Teile wechseln deutlich Abfälle mit Perioden einer gewissen Konstanz der Werte, sodaß sich die Kurve wellenförmig um die supponierte Gerade herumwindet. Immerhin bietet die ganze Kurve ein Bild, welches der Interferenz von zweierlei Zersetzungsprozessen entspricht, und zwar von einem sehr rasch und von einem recht langsam vor sich gehenden. Um nur wieder eine Vorstellung darüber zu entwickeln, wäre als Beispiel eines raschen Zersetzungsprozesses die Zerlegung der Verbindung von Chlorwasserstoff an säureamidartig gebundenen Stickstoff der eiweißähnlichen Stoffe anzusehen, als Beispiel eines recht langsamen Zersetzungsprozesses die Abgabe von Chlorwasserstoff aus dessen Reaktionsprodukt mit unorganischen Stoffen (z. B. Phosphaten).

Aus den Resultaten derjenigen Versuche, bei welchen unter Erwärmung auf $50^{\circ} \mathrm{C}$. Luft übergeleitet worden war, ist früher ein gewisser Ruhepunkt der Zersetzung herausgelesen worden. Sucht man nun die diesen Resultaten entsprechenden Werte in der prozentischen Tabelle der Auspumpversuche auf, (sie sind dort mit * bezeichnet worden), so sieht man, daß dieser Punkt keineswegs den Endpunkt der Zersetzung bedeutet, weil in den Auspumpversuchen von diesem Punkte an die Werte noch weiter fallen. Er kann aber insofern als ein Ruhepunkt betrachtet werden, als bei den Auspumpversuchen an dieser Stelle die Werte durch einige Zeit ziemlich konstant bleiben und nicht viel abnehmen. 


\section{Bestimmung der Acidität.}

In derselben Absicht, wie bei den Diastaseversuchen, wurde auch an den mit Chlorwasserstoff behandelten Invertasepräparaten die Acidität durch Titration mit 1/5-NormalBarytwasser gegen Phenolphthalein durchgeführt. An diese Bestimmung wurde gleich die Formoltitrierung angeschlossen. Die Details dieser Versuche sind genau dieselben wie dort. Die beiden letzten Versuche der folgenden Tabelle sind mit dem Materiale der Auspumpversuche II und III angestellt.

\begin{tabular}{|c|c|c|c|}
\hline \multirow{2}{*}{$\begin{array}{l}\text { Verwendete Menge } \\
\text { des } \\
\text { Invertasepräparates } \\
\mathrm{g}\end{array}$} & \multirow[b]{2}{*}{$\begin{array}{l}\text { Chlorwasser- } \\
\text { stoffgehalt } \\
\text { g }\end{array}$} & \multicolumn{2}{|c|}{ Normallauge verbraucht } \\
\hline & & $\begin{array}{c}\text { zur } \\
\text { Neutralisation } \\
\text { ccm }\end{array}$ & $\begin{array}{c}\text { zur } \\
\text { Formoltitrierung } \\
\mathrm{ccm}\end{array}$ \\
\hline 0,5320 & 0,1163 & 4,000 & 0,790 \\
\hline 0,4940 & 0,1068 & 3,694 & 0,855 \\
\hline \multicolumn{4}{|c|}{ Auspumpversuche: } \\
\hline 0,4873 & 0,0697 & 2,764 & 0,895 \\
\hline 0,4698 & 0,0737 & 2,989 & 0,786 \\
\hline
\end{tabular}

In einer weiteren Tabelle berechne ich diese Werte in derselben Weise wie bisher prozentisch, ich berechne weiter daraus die Aciditätszunahme als "gefundene Aciditätszunahme» und stelle diesen Zahlen die aus dem Chlorwasserstoffgehalte berechnete als «berechnete Aciditätszunahme» gegenüber.

\begin{tabular}{c|c|c|c|c}
\hline \hline \multirow{2}{*}{$\begin{array}{c}\text { Chlorwasser- } \\
\text { stoffgehalt }\end{array}$} & $\begin{array}{c}\text { Acidität } \\
\text { des behandelten } \\
\text { Präparates } \\
\text { ccm }\end{array}$ & $\begin{array}{c}\text { des ursprünglichen } \\
\text { Präparates } \\
\text { ccm }\end{array}$ & gefunden & berechnet \\
ccm & ccm \\
\hline 21,86 & 751,9 & 160,2 & 591,7 & 599,4 \\
21,62 & 747,8 & 160,2 & 587,6 & 592,9 \\
14,30 & 567,2 & Auspumpversuche: & & \\
15,69 & 636,2 & 160,2 & 407,0 & 392,1 \\
& & 160,2 & 476,0 & 430,3
\end{tabular}

In den beiden ersten Versuchen stimmt die gefundene Aciditätszunahme mit der berechneten recht gut überein. In 
den Auspumpversuchen ist sogar die gefundene Aciditätszunahm̌e größer als die berechnete. Aus weiteren Betrachtungen wird sich ergeben, daß bei den Auspumpversuchen der Chlorwasserstoffgehalt und damit die berechnete Aciditätszunahme einer Korrektur bedarf. Bringt man nun diese Korrektur an, so erhöht sich der Chlorwasserstoffgehalt auf 15,07\%, bezw. $16,76 \%$ und damit die berechnete Aciditätszunahme auf $413,3 \mathrm{ccm}$, bezw. 459,6 ccm, und damit ist eine befriedigende Übereinstimmung mit der gefundenen Aciditätszunahme gegeben.

Mit und ohne Korrektur aber läßt sich aus dieser Gegenüberstellung die Existenz einer Gruppe, welche in anderer Weise denn als Ionenreaktion Ghlorwasserstoffchemie binden würde, nicht ersehen. Es bedeutet dies einen hervorzuhebenden Unterschied gegenüber den Diastasepräparaten.

Fomoltitrierungen.

Die bereits angeführten Resultate der Formoltitrierungen geben in der wie bisher durchgeführten prozentischen Berechnung folgende Werte:

Versuche ohne Auspumpen: 2,08\%, bezw. 2,42\%.

Auspumpversuche: $\quad 2,57 \%, \quad>2,34 \%$.

Diese Zahlen sind durchwegs kleiner als die Zahlen, welche bei der Formoltitrierung des unveränderten Invertasepräparates gefunden worden sind $(3,25 \%$, bezw. 3,09\%, im Mittel: $3,17 \%$ ). Es ist demnach durch die Einwirkung von Chlorwasserstoff formoltitrierbarer Stickstoff in andere Bindung übergeführt worden. Offenbar liegt dieser anderen Bindung eine Anhydridbildung zugrunde, indem freie Carboxylgruppen unter der Einwirkung des Ghlorwasserstoffs mit freien Aminogruppen säureamidartige Bindungen unter $\mathrm{Ab}$ spaltung von Wasser eingegangen sind.

Nun ist zweifellos bei den Auspumpversuchen dieses abgespaltene Wasser aus den Präparaten bei deren 20 tägigem Verweilen im Vakuum über Schwefelsäure und Natronkalk entfernt worden. Das Plus, welches deren Gewicht nach dem Auspumpen gegenüber ihrem Gewichte von der Chlorwasser- 
stoffbehandlung zeigt, entspricht daher nicht genau dem Chlorwasserstoffgehalte, sondern es bedeutet die Differenz zwischen dem Chlorwasserstoffgehalte und dem Gewichte des abgegebenen Wassers. Das Gewicht des abgegebenen Wassers läßt sich nun leicht berechnen, indem jedem verschwundenen Atom formoltitrierbaren Stickstoffs ein Molekül Wasser entspricht. Die so gefundene Zahl muß nun dem scheinbaren Chlorwasserstoffgehalte hinzugerechnet werden, wenn man den wirklichen Chlorwasserstoffgehalt ermitteln will. Das ist die Korrektur, welche bei den Auspumpversuchen im vorigen Abschnitt angebracht worden ist.

Die Entscheidung der Frage, ob dieselbe Korrektur auch bei den nicht ausgepumpten Präparaten anzubringen sei, würde davon abhängen, ob das abgespaltene Wasser aus dem Präparate entfernt worden ist. Darüber könnte man aber höchstens unbegründete Vermutungen hegen. Die Frage muß also dahingestellt werden. Es bleibt demnach der im vorigen Abschnitt gezogene Schlußsatz aufrecht.

Bestimmung des Amidstickstoffes.

Sie wurde in genau derselben Weise durchgeführt wie bei den Diastasepräparaten und lieferte in analoger Darstellung folgende Resultate:

$0,5103 \mathrm{~g}$ nahmen auf $0,1173 \mathrm{~g}=22,99 \%$ Chlorwasserstoff und lieferten $19,9 \mathrm{~cm} \mathrm{~N}$ bei $20,0^{\circ} \mathrm{C}$. und $742,8 \mathrm{~mm}$, entsprechend 2,18\% Amidstickstoff.

$0,4914 \mathrm{~g}$ nahmen auf $0,1009 \mathrm{~g}=20,53 \%$ Chlorwasserstoff und lieferten $15,0 \mathrm{ccm} \mathrm{N}$ bei $20,1^{\circ} \mathrm{C}$. und $744,7 \mathrm{~mm}$, entsprechend 1,71\% Amidstickstoff.

Auch hier ergeben sich niedrigere Zahlen wie bei der Bestimmung des Amidstickstoffes an dem unveränderten Invertasepräparat. Es wird also dadurch das Resultat der Formoltitrierungen vollauf bestätigt.

\section{Prüfung der Fermentwirkung.}

Zur Prüfung der mit Chlorwasserstoff behandelten Fermentpräparate auf ihre Wirksamkeit (es waren bei allen Ver- 
suchen je $0,2 \mathrm{~g}$ des Invertasepräparates in Arbeit genommen worden) wurden diese Präparate mit etwas Wasser übergossen und sofort mit einer berechneten Menge Zehntel-Normallauge versetzt. Nachdem sich das Präparat vollständig aufgelöst hatte, wurde die Lösung auf $25 \mathrm{ccm}$ aufgefüllt und blieb so eine Stunde lang stehen. Die Menge der Zehntel-Normalnatronlauge wurde aus dem Chlorwasserstoffgehalte des Präparates derart berechnet, daß nach dem Zusatze der Lauge noch eine Chlorwasserstoffmenge übrig blieb, welche $0,25 \mathrm{ccm}$ ZehntelNormalsalzsäure entsprach.

Zur Kontrolle diente eine Lösung von 0,2 g des Invertasepräparates, welche mit $0,25 \mathrm{ccm}$ Zehntel-Normalsalzsäure versetzt, auf $25 \mathrm{ccm}$ aufgefüllt war und dann eine Stunde lang stehen blieb.

Nach dieser Zeit wurden die Lösungen mit je $50 \mathrm{ccm}$ einer auf $40^{\circ}$ C. erwärmten $20 \%$ igen Rohrzuckerlösung vermengt und in ein auf $40^{\circ} \mathrm{C}$. erwärmtes Wasserbad eingesetzt. Gleich bei Beginn, sowie nach angemessenen Zeiträumen wurden dem Gemenge je $10 \mathrm{ccm}$ entnommen und sofort mit je $10 \mathrm{ccm} \mathrm{0,2} \mathrm{Normal-Natronlauge} \mathrm{vermengt.} \mathrm{Die} \mathrm{dadurch} \mathrm{sich}$ leicht trübende Flüssigkeit wurde filtriert und das Filtrat im $2 \mathrm{dm}$-Rohre im Polarisationsapparate untersucht. Der verwendete Polarisationsapparat zeigte direkt Prozente Traubenzucker an. Die abgelesene Zahl ist ohne weitere Umrechnung in die folgenden Tabellen eingesetzt. Ich führe von mehreren gleichartigen Versuchen nur zwei an.

\begin{tabular}{|c|c|c|c|}
\hline $\begin{array}{c}\text { Dauer } \\
\text { des Versuches }\end{array}$ & $\begin{array}{l}\text { Unverändertes } \\
\text { Invertasepräparat }\end{array}$ & \multicolumn{2}{|c|}{$\begin{array}{l}\text { Mit Chlorwasserstoff behandelte } \\
\text { Invertasepräparate }\end{array}$} \\
\hline Beginn & $+8,2$ & $+8,5$ & $\begin{array}{r}7,7 \\
\end{array}$ \\
\hline 1/4 Stunde. & $+7,3$ & $+8,3$ & $+7,6$ \\
\hline $1 / 2>\quad$ & $+6,4$ & $+8,3$ & $+7,6$ \\
\hline 3/4 Stunden . & $+5,6$ & $+8,3$ & $+7,6$ \\
\hline 1 Stunde.. & $+5,0$ & $+8,3$ & $+7,6$ \\
\hline $11 / 4$ Stunden . & $+4,1$ & $+8,2$ & $+7,7$ \\
\hline $11 / 2$, & $+3,4$ & $+8,3$ & $+7,7$ \\
\hline
\end{tabular}


In ähnlicher Weise verlief ein Versuch, welcher mit dem Präparate vom Auspumpversuche I angestellt wurde.

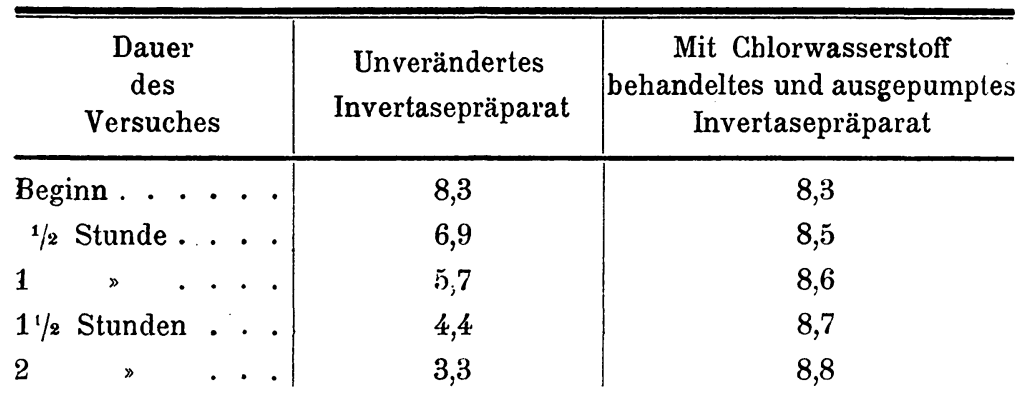

Durch die Einwirkung von Chlorwasserstoff wird demnach die Wirksamkeit der Invertase vernichtet, und sie kehrt beim Auspumpen nicht wieder.

Die Vernichtung der Wirksamkeit der Invertase kann in meinen Versuchen nicht darauf beruhen, daß der Chlorwasserstoff als Säure mit basischen Gruppen der Invertase salzartige Verbindungen eingeht, weil die Wirksamkeit nicht wiederkehrt, wenn man den aufgenommenen Chlorwasserstoff durch Lauge neutralisiert. Atomgruppen, welche Chlorwasserstoff in anderer Weise chemisch binden können, sind, namentlich in den Auspumpversuchen, nicht aufgefunden worden (siehe Aciditätsbestimmung). Die Vernichtung der Fermentwirkung kann daher auch nicht auf die Festlegung solcher Atomgruppen durch deren Verbindung mit Chlorwasserstoff zurückgeführt werden.

Dagegen wurde eine durch die Einwirkung des Chlorwasserstoffs verursachte Abnahme des formoltitrierbaren Stickstoffs, bezw. Amidstickstoffs konstatiert, welche auf eine säureamidartige Verbindung von Carboxylgruppen mit Aminogruppen hindeutet.

Es liegt nun nahe, diese chemische Reaktion für die Vernichtung der Fermentwirkung verantwortlich zu machen. Damit verlasse ich aber realen Boden und begebe mich auf hypothetisches Gebiet; hypothetisch darum, weil nicht erwiesen ist, daß gerade diese chemische Reaktion die Ursache der Auf- 
hebung der Fermentwirkung ist. Es könnte ja auch daneben noch eine andere chemische Reaktion vor sich gegangen sein, die nicht konstatiert wurde und die keine Ionenreaktion ist. Eine solche Reaktion könnte z. B. die Polymerisation von Aldehyden sein, vorausgesetzt, daß die Invertase Aldehydgruppen enthält.

Ist aber die genannte Anhydridbildung wirklich die Ursache der Vernichtung der Fermentwirkung, dann ist auch gewiß der hohe Gehalt des Fermentpräparates an formoltitrierbarem Stickstoff $(3,17 \%)$ gegenüber der Gesamtstickstoffmenge $(5,57 \%)$ nicht belanglos. Das Verhältnis ändert sich aber noch zugunsten des formoltitrierbaren Stickstoffes. wenn man bedenkt, daß das Fermentpräparat noch Eiweißreaktionen zeigt, daß bekanntermaßen die die Eiweißreaktionen gebenden Stoffe zur invertierenden Wirkung nicht notwendig sind, und daß die Eiweißstoffe im Vergleiche zu ihrem gesamten Stickstoffgehalte nur recht wenig formoltitrierbaren Stickstoff enthalten. Man kann demnach sagen, daß in dem wirksamen Anteile des Fermentpräparates die Menge des formoltitrierbaren Stickstoffes größer ist als die Menge des in anderer Weise gebundenen Stickstoffes.

Einem solchen Verhältnisse würde entsprechen ein Gemenge von Stoffen, in welchem nebst anderen, insbesondere sauer reagierenden Stoffen, größere Mengen von Salzen primärer Amine enthalten sind, oder aber ein Gemenge von Aminosäuren mit voraussichtlich recht einfach gebauten Polypeptiden. Man könnte sogar in dem Umstande, daß die Zahlen für den Amidstickstoff $(2,30 \%)$ erheblich kleiner sind als die Zahlen für formoltitrierbaren Stickstoff $(3,17 \%)$, obwohl man nach der Ausführung der Versuche (lange Dauer der Einwirkung der salpetrigen Säure) sogar höhere Zahlen hätte erwarten sollen, einen Hinweis auf jene Aminosäuren erblicken, welche bei der Behandlung mit salpetriger Säure nicht ihren ganzen Stickstoff in Freiheit setzen.

Ich habe mit diesen Sätzen eine Arbeitshypothese aufgestellt, deren Richtigkeit durch spätere Versuche eingehend zu prüfen sein wird. 
390 Th. Panzer, Einwirkung von Chlorwasserstoff auf Invertase II.

Als bewiesenes Ergebnis meiner Versuche aber möhte ich hinstellen, daß die Vernichtung der Fermentwirkung dirch Einwirkung von gasförmigem Chlorwasserstoff bei der Inverase auf anderen chemischen Prozessen beruht als bei der urtersuchten Diastase, und schließe daraus, daß auch die chemiche Konstitution des wirksamen Anteiles der Invertase eine ancere ist als die des wirksamen Anteiles der untersuchten Diastise. 